\title{
II DBS come test diagnostico nella malattia di Fabry
}

\author{
Simone Scalia \\ Centro di Ricerca e Diagnosi Malattie da Accumulo Lisosomiale, Istituto di Biomedicina e Immunologia Molecolare "A. Monroy" (IBIM), Consiglio \\ Nazionale delle Ricerche (CNR), Palermo
}

\begin{abstract}
DBS assay in the diagnosis of Fabry disease
Fabry disease (FD) is a disorder caused by the functional deficit of $\alpha$-galactosidase A. Therefore, one of the diagnostic tests for FD is the study of enzymatic activity. This analysis can be performed on plasma, isolated leukocytes or dried blood spot (DBS). The DBS assay is the most widely used method today because it is reliable, fast, cheap and relatively easy to perform. Since 10 years, in the Research Center on Metabolic Diseases of the IBIM-CNR of Palermo, the DBS assay has been used to evaluate the $\alpha$-galactosidase A activity in patients with signs and symptoms referable to FD. The assay was optimized to make it more reliable. In a retrospective study, we analyzed the enzymatic activity in 200 individuals affected by the classic form of FD: 100\% of the male patients showed null activity, while only $43 \%$ of the female patients showed abnormal values. If we had perfomed the DBS assay as the only diagnostic test, $57 \%$ of women would not have received the proper diagnosis. Indeed, it is known that affected females can show normal enzyme activity due to inactivation of the X-chromosome. For this reason, the DBS assay is currently considered the main assay for FD diagnosis in males, while genetic analysis is the most reliable test to confirm the clinical suspicion of FD in women.
\end{abstract}

Keywords: Alpha-galactosidase A, DBS test, Fabry disease

\section{I test enzimatici per la diagnosi di malattia di Fabry}

La malattia di Fabry $(\mathrm{mF})$ è una patologia causata dal deficit funzionale dell' $\alpha$-galattosidasi A ( $\alpha$-Gal A) (1). Per questo motivo, uno dei test diagnostici maggiormente utilizzati in pazienti con sospetto clinico di $\mathrm{mF}$ è lo studio dell'attività enzimatica. Ad oggi, tale attività può essere valutata con tre diverse metodiche: la prima, effettuata su plasma, rappresenta uno dei saggi maggiormente diffusi nel mondo per la sua relativa facilità di realizzazione $(2,3)$. Tuttavia, tale analisi richiede particolare attenzione nei tempi di esecuzione (l'attività decade in poche ore), nella preparazione dei campioni, nell'uso di un tampone a elevata forza ionica e nella presenza di fattori plasmatici che possono disturbare la determinazione. Per queste ragioni, le attività ottenute in questo modo non sempre forniscono dei risultati affidabili e ripetibili.

Accepted: July 15, 2017

Published online: July 28, 2017

Indirizzo per la corrispondenza:

Dr. Simone Scalia

Centro di Ricerca e Diagnosi

Malattie da Accumulo Lisosomiale

Consiglio Nazionale delle Ricerche

Istituto di Biomedicina e Immunologia Molecolare "A. Monroy"

Via Ugo La Malfa, 153

90146 Palermo

simone.scalia@ibim.cnr.it
La seconda metodica si basa nel valutare l'attività dell'enzima sui leucociti isolati (3). Tale metodologia, basata sulla purificazione di queste cellule dal sangue e successivo dosaggio delle proteine totali, fornisce dei valori attendibili e affidabili. Tuttavia, tale analisi risulta di difficile realizzazione, a causa dei lunghi tempi di preparazione (4) e di una quantità di sangue di partenza maggiore rispetto agli altri metodi. Per questi motivi, in molti laboratori questa metodica non viene sempre utilizzata come test diagnostico di routine.

il terzo metodo, chiamato Dried Blood Spot (DBS) test, prevede l'analisi enzimatica su spot di sangue essiccato (5). Rispetto agli altri saggi, questo metodo presenta diversi vantaggi come l'elevata stabilità dell'enzima nelle spot, per diverse settimane, la bassa quantità di sangue di partenza, la rapidità e l'affidabilità dell'analisi. Perciò, oggi il DBS è il test più utilizzato nel mondo per lo studio dell'attività dell' $\alpha$-Gal $A$, e la bontà di tale metodica è stata confermata dal lavoro di Scott et al (6), in cui gli autori ne hanno valutato e dimostrato la validità tramite saggi di spettrometria di massa.

\section{L'esperienza dell'IBIM-CNR}

Da oltre dieci anni, presso il Centro di Ricerca sulle Malattie Metaboliche dell'IBIM-CNR, viene utilizzato il DBS test come principale metodo diagnostico per saggiare l'attività $\alpha$-Gal A. Il saggio è stato da noi ottimizzato per essere ancora più affidabile: nei nostri laboratori il test viene realizzato su piastre da 96 pozzetti, invece che in tubi da $1 \mathrm{~mL}$ come inizialmente proposto da Chamoles et al (5), consentendo lo studio 
in modo più rapido; il saggio viene realizzato su spot di sangue essiccato di dimensioni maggiori, rispetto al protocollo originale, 6 millimetri invece di 3, consentendo una migliore distribuzione del sangue sulla spot e quindi una maggiore riproducibilità dei risultati; per ogni campione, viene valutato il rumore di fondo tramite una reazione effettuata in presenza del DGJ, una molecola capace di inibire l'attività dell' $\alpha$-Gal A quando presente a concentrazioni elevate. Conoscere il rumore di fondo è essenziale per calcolare l'esatta attività enzimatica di un paziente, rendendo tale metodica più precisa; in ogni piastra vengono aggiunti sempre un controllo positivo e un controllo negativo per saggiare la bontà di ogni esperimento. Inoltre, in ogni saggio viene realizzata una curva di calibrazione, in modo da eliminare la variabilità inter-piastra intrinseca alla metodica stessa. In tutti questi anni, il team di ricerca del Professor Duro ha studiato circa 15.000 soggetti con segni e sintomi riconducibili alla $\mathrm{mF}$, identificando oltre 350 soggetti con mutazioni esoniche in GLA, di cui 200 affetti dalla forma classica della malattia. Vista la grande casistica di pazienti, abbiamo effettuato uno studio retrospettivo su questi 200 soggetti; il 100\% dei maschi affetti mostrava un'attività enzimatica patologica, mentre soltanto il $43 \%$ delle donne affette presentava dei valori patologici. Questo è dovuto alla lyonizzazione, inattivazione casuale di uno dei 2 cromosomi $X$, un fenomeno descritto per la prima volta nel 1961 da Mary Lyon. La lyonizzazione è un normale processo biologico che interessa tutte le femmine di mammifero. Durante lo sviluppo embrionale uno dei 2 cromosomi $X$ in ciascuna cellula somatica diviene trascrizionalmente inattivo, ma l'inattivazione non coinvolge lo stesso cromosoma in tutte le cellule dell'organismo. Nelle cellule di un organo può essere attivato quello paterno nelle cellule di un altro organo potrebbe essere attivato quello materno. A causa di questo fenomeno, le donne, di solito eterozigoti, sono un "mosaico" di cellule normali e malate. Quando si effettua l'analisi dell'attività enzimatica, in una donna affetta da $\mathrm{mF}$, è possibile rilevare dei valori nella norma derivanti da quel pool di cellule normali, non consen- tendo quindi una corretta diagnosi della malattia con il semplice studio dell'attività enzimatica (questo a prescindere dal metodo utilizzato). Per tale motivo, ad oggi l'analisi genetica rappresenta il principale test diagnostico per la $\mathrm{mF}$ nelle donne. In conclusione il DBS rappresenta il saggio d'elezione per la conferma del sospetto clinico nei soggetti maschi con una sintomatologia riconducibile alla Fabry. Questa metodica, con la sua rapidità e affidabilità, fornisce delle risposte diagnostiche certe in tempi brevi, consentendo ai pazienti di iniziare la terapia il più precocemente possibile.

\section{Disclosures}

Financial support: No financial support was received for this submission.

Conflict of interest: The author has no conflict of interest.

\section{Bibliografia}

1. Brady RO, Gal AE, Bradley RM, Martensson E, Warshaw AL, Laster L. Enzymatic defect in Fabry's disease. Ceramidetrihexosidase deficiency. N Engl J Med. 1967;276(21):1163-7.

2. Froissart R, Guffon N, Vanier MT, Desnick RJ, Maire I. Fabry disease: $\mathrm{D} 313 \mathrm{Y}$ is an alpha-galactosidase $\mathrm{A}$ sequence variant that causes pseudodeficient activity in plasma. Mol Genet Metab. 2003;80(3):307-14.

3. Desnick RJ, Allen KY, Desnick SJ, Raman MK, Bernlohr RW, Krivit W. Fabry's disease: enzymatic diagnosis of hemizygotes and heterozygotes. Alpha-galactosidase activities in plasma, serum, urine, and leukocytes. J Lab Clin Med. 1973;81(2):157-71.

4. Daitx VV, Mezzalira J, Goldim MP, Coelho JC. Comparison between alpha-galactosidase $\mathrm{A}$ activity in blood samples collected on filter paper, leukocytes and plasma. Clin Biochem. 2012;45(15):1233-8.

5. Chamoles NA, Blanco M, Gaggioli D. Fabry disease: enzymatic diagnosis in dried blood spots on filter paper. Clin Chim Acta. 2001;308(1-2):195-6.

6. Scott CR, Elliott $S$, Buroker $N$, et al. Identification of infants at risk for developing Fabry, Pompe, or mucopolysaccharidosis-I from newborn blood spots by tandem mass spectrometry. $J$ Pediatr. 2013;163(2):498-503. 